\title{
Avaliação ocular multimodal em doenças heredodistróficas e degenerativas da retina
}

\author{
Multimodal fundus imaging in heredodystrophic \\ and degenerative diseases of the retina
}

Daniela Cavalcanti Ferrara ${ }^{1}$, Daniela Calucci ${ }^{2}$, Juliana Lambert Oréfice ${ }^{2}$, Rogério Alves Costa ${ }^{1,2,3}$

\section{Resumo}

A tomografia de coerência óptica incorporou-se gradativamente ao contemporâneo arsenal diagnóstico em Oftalmologia, passando a exercer papel fundamental na investigação e condução de doenças oculares, particularmente na especialidade de Retina e Vítreo. A disponibilização comercial da nova geração de aparelhos, chamada de tomografia de coerência óptica "espectral", baseada em conceito físico distinto que permite a aquisição de imagens em alta velocidade, marcou o início de uma nova era desta tecnologia de investigação auxiliar. Adicionalmente, sua recente combinação com o oftalmoscópio de varredura a laser confocal (confocal scanning laser ophthalmoscope) vem propiciando a aquisição de imagens tomográficas guiadas em tempo real pelos diferentes modos de imagem (autofluorescência de fundo, reflectância com luz "infravermelha" e angiografia com fluoresceína ou indocianina verde). A avaliação ocular multimodal (multimodal fundus imaging) permite a correlação real e minuciosa de achados da morfologia retiniana e do epitélio pigmentar com dados de estudos angiográficos e de autofluorescência ou reflectância, propiciando assim inferências valiosas sobre a fisiologia do tecido. Neste artigo, discutimos brevemente as possíveis implicações da avaliação ocular multimodal na prática da especialidade de Retina e Vítreo.

Descritores: Angiografia; Epitélio pigmentado da retina; Tomografia de coerência óptica/métodos; Fluorescência; Espectroscopia infravermelho transformada de Fourier; Laser; Retina

\footnotetext{
'Departamento de Oftalmologia, Otorrinolaringologia e Cirurgia de Cabeça e Pescoço, Faculdade de Medicina de Ribeirão Preto da Universidade de São Paulo - USP - Ribeirão Preto (SP), Brasil.

${ }^{2}$ Divisão de Mácula do Centro Brasileiro de Ciências Visuais - Belo Horizonte (MG), Brasil.

${ }^{3}$ Programa de Pós-graduação em Ciências Aplicadas à Cirurgia e à Oftalmologia, Universidade Federal de Minas Gerais - UFMG Belo Horizonte (MG), Brasil.

Recebido para publicação em: 24/9/2009 - Aceito para publicação em 18/10/2009
} 


\section{INTRODUÇÃO}

A investigação fenotípica das doenças heredodistróficas e degenerativas da retina e epitélio pigmentado (EPR) tem sido, há muito, baseada em métodos de imagem como a oftalmoscopia e retinografia bem como estudos angiográficos e eletrorretinográficos. ${ }^{1} \mathrm{Na}$ última década, porém, novas modalidades de imagem como a autofluorescência de fundo (fundus autofluorescence, FAF), a documentação com luz "infravermelha" (near-infrared reflectance, $n I R$ ) e a tomografia de coerência óptica (optical coherence tomography, $O C T$ ), vêm se incorporando ao arsenal investigativo auxiliar utilizado na caracterização morfológica e funcional da retina e EPR.

Os sistemas de documentação fotográfica/ angiográfica em retina dividem-se basicamente em câmera de fundo convencional ("retinógrafo") e o oftalmoscópio de varredura a laser confocal (confocal Scanning Laser Ophthalmoscope, cSLO). Enquanto na primeira flashes de luz branca com filtros específicos são utilizados na excitação dos tecidos/contrastes endovenosos (fluoresceína ou indocianina verde), lasers no comprimento de onda exato são empregados no $c S L O$ na excitação dos pigmentos, sejam estes endógenos ou exógenos. Mais recentemente, avanços tecnológicos nesta área culminaram na geração de sistemas com capacidade de aquisição simultânea de imagens em diferentes modos. Alguns aparelhos de $c S L O$, por exemplo, possibilitam a aquisição de imagens tomográficas de alta-resolução em alta-velocidade guiadas em tempo real pelos diferentes modos de imagem disponíveis ( $n I R, F A F$, angiografia com fluoresceína ou indocianina verde) (Figura 1). ${ }^{1}$ A correlação real e pontual de achados da morfologia retiniana e do EPR com dados de estudos angiográficos e/ou $F A F$ e $n I R$ vem propiciando inferências valiosas sobre a fisiologia ocular in vivo..$^{1-5}$ A seguir, discutiremos brevemente alguns aspectos dos diferentes modos que caracterizam esta nova modalidade diagnóstica, chamada de "avaliação ocular multimodal" (multimodal fundus imaging) (Figura 2) ${ }^{1-3}$

Tomografia de Coerência Óptica de Alta-Resolução e Alta-Velocidade (High-Resolution High-Speed Optical Coherence Tomography)

Duas mudanças essenciais marcam a nova geração de aparelhos de $O C T$ disponibilizada recentemente: 1) o emprego de fontes de luz ( $S L D s)$ de banda espectral mais larga, e 2) a adoção da tecnologia "Fourier-domain/ espectral" para processamento dos sinais.
A largura da banda espectral da fonte de luz $(S L D s)$ do $O C T$ está diretamente relacionada à resolução (axial) do aparelho; simplisticamente, quanto maior a largura da banda, maior a resolução axial. A largura da banda espectral da fonte de luz dos novos aparelhos aumentou consideravelmente em relação à terceira geração (Stratus OCT), com conseqüente aumento em sua

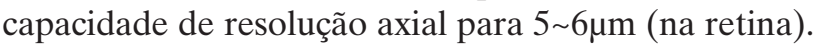
Já a resolução transversal (lateral), que sofre influência de fatores adicionais como, por exemplo, a qualidade da parte óptica do aparelho, ficou entre 14 20 $\mu$ m nesta nova geração. Com estas características, esta geração de tomógrafos foi classificada como high-resolution (altaresolução).

Para a geração de imagens bidimensionais ( $B$-scan ou tomograma) da retina (principalmente região macular e peridiscal), os aparelhos de $O C T$ realizam análise individual de vários $A$-scans que compõe um determinado tomograma. Consequentemente, a "definição" (ñ̃a "resolução") de um tomograma (B-scan) é influenciada diretamente pelo número de $A$-scans que compõe esta imagem, bem como a capacidade do software em identificar (e eliminar) "ruídos" e artefatos (Figura 3). Os aparelhos antigos utilizavam um princípio básico de funcionamento chamado time-domain que, por razões técnicas (movimentação do espelho de referência), limitava a capacidade de geração de $A$-scans em $400 A$-scans por segundo. Assim, 1.28 segundo era necessário para a captura de um tomograma de alta-densidade (512 A-scans) no aparelho de terceira geração. Os novos tomógrafos adotam o princípio Fourier-domain para o processamento dos sinais, saindo de cena o "espelho de referência" e entrando o "espectrômetro" (razão da terminologia "spectral OCT"). Essa substituição trouxe mudanças profundas na capacidade de geração de $A$-scans pelos novos tomógrafos, elevando as taxas para até $40.000 A$-scans por segundo (aumento de 100 vezes) e possibilitando a aquisição de dados suficientes para reconstruções tridimensionais do tecido estudado (Figura 4).

Todos os novos aparelhos disponíveis no presente momento utilizam tanto fontes de luz de banda espectral mais larga (maior resolução) bem como o princípio Fourier-domain de processamento dos sinais (maior capacidade de geração de $A$-scans); são, portanto, aparelhos com capacidade de imagem em "alta-resolução" e "alta-velocidade" ${ }^{6,7} \mathrm{Na}$ prática, essa nova tecnologia permite melhor individualização das camadas retinianas e caracterização da interface vitreorretiniana (Figura 5), capaz de revelar peculiaridades morfológicas não detectáveis pela semiologia clínica rotineira. ${ }^{6-10}$ 


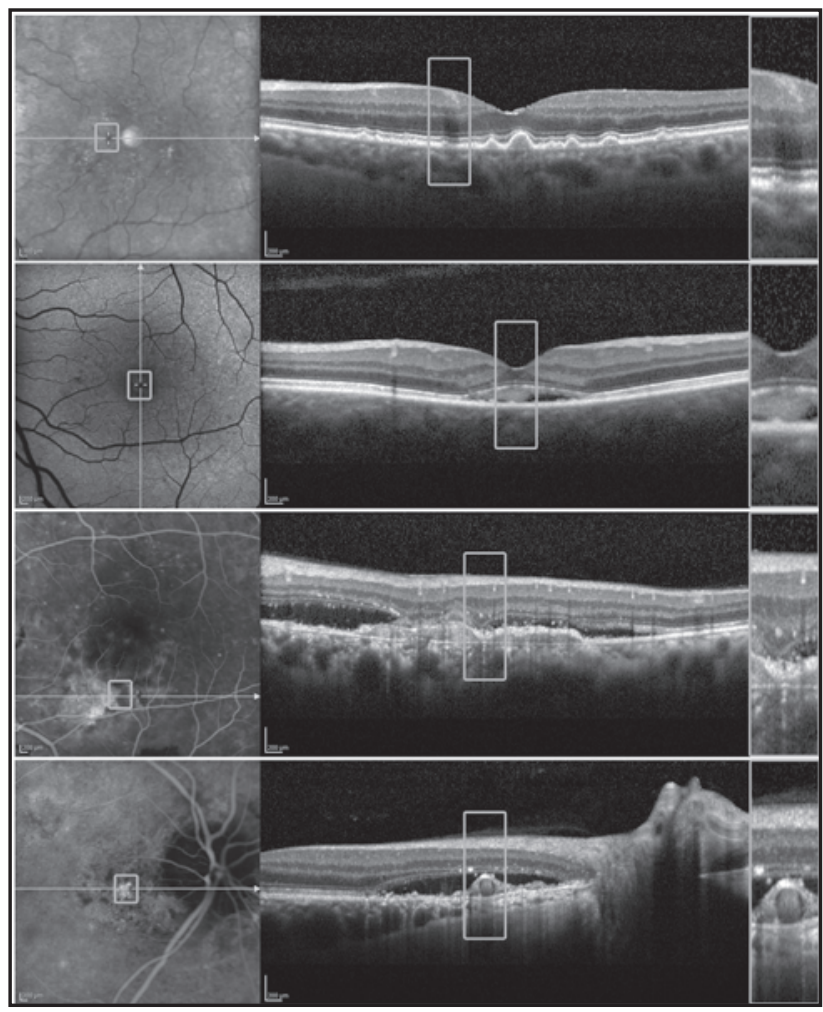

Figura 1. Exemplos de avaliação ocular multimodal em doenças da retina e coróide, com a combinação da tomografia de coerência óptica espectral com diferentes modos (reflectância com luz "infravermelha", autofluorescência de fundo, angiografia com fluoresceína e angiografia com indocianina verde [superior para inferior, respectivamente]).

\section{Autofluorescência de Fundo \\ (Fundus Autofluorescence, FAF)}

A documentação da autofluorescência do fundo ocular permite a inferência de características funcionais do EPR (e indiretamente da retina) por documentar de forma não-invasiva a presença de lipofuscina no tecido. ${ }^{11-17}$ Durante a aquisição de $F A F$ com o $c S L O$ a retina é iluminada com comprimento de onda de $488 \mathrm{~nm}$, e o filtro de barreira é idêntico ao utilizado para angiografia com fluoresceína. ${ }^{17}$ Como essencialmente apenas as estruturas com lipofuscina são registradas, a interpretação dos achados de $F A F$ depende da interação dos sinais autofluorescentes oriundos do EPR com estruturas retinianas (normais ou patológicas) sobrejacentes. ${ }^{11,12,18}$

A lipofuscina é uma mistura de várias biomoléculas, incluindo diferentes fluoróforos, e seu principal constituinte foi identificado como A2-E (Nretinildeno N-retiniletanolamina). ${ }^{19-22}$ É pigmento autofluorescente que se acumula na porção basal da célula do EPR, como bioproduto da fagocitose dos segmentos externos dos fotorreceptores, sendo considerado um marcador do envelhecimento celular fisiológico. ${ }^{23}$
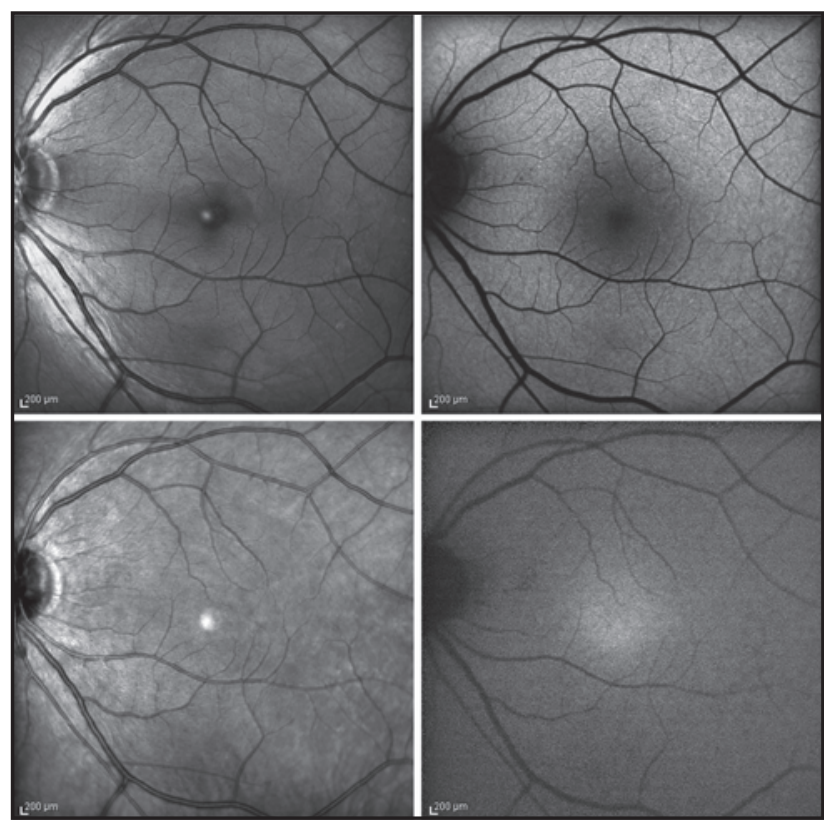

Figura 2. Avaliação ocular multimodal dentro dos limites da normalidade utilizando oftalmoscópio de varredura a laser confocal (Spectralis ${ }^{\circledR}$ HRA+OCT; Heidelberg Engineering Inc., Heidelberg, Alemanha). A retinografia red-free (superior esquerda), documenta as estruturas mais superficiais. A autofluorescência de fundo (fundus autofluorescence, $F A F$ ) (superior direita) registra estruturas com lipofuscina. A reflectância com luz "infravermelha" (near-infrared reflectance, $n I R$ ) (inferior esquerda) permite a documentação de estruturas retinianas profundas e sub-retinianas. A autofluorescência com luz "infravermelha" (near-infrared autofluorescence, $n I A$ ) (inferior direita) está teoricamente relacionada a distribuição de melanina.

Entretanto, os segmentos externos dos fotorreceptores contém proteínas, retinóides e grandes proporções de ácidos graxos poliinsaturados suscetíveis ao dano fotooxidativo, e o acúmulo anormal de lipofuscina tem sido diretamente associado ao comprometimento de funções metabólicas essenciais do EPR. Os variados mecanismos de dano ao EPR incluem inibição da capacidade degradativa lisossomal, perda da integridade de membrana celular, e fototoxicidade. ${ }^{24-31}$

Evidências epidemiológicas, clínicas e experimentais indicam que o acúmulo de lipofuscina no EPR precede a atrofia das camadas celulares da retina externa, incluindo degeneração de fotorreceptores e atrofia do próprio EPR. ${ }^{26,30,32-34}$ A perda da função visual também em patologias heredodistróficas como Doença de Stargardt e Doença de Best tem sido atribuída ao acúmulo anormal de lipofuscina no EPR e/ou no espaço sub-retiniano. ${ }^{12,18,35-}$ ${ }^{37}$ A FAF mostrou-se de grande utilidade nestas doenças, assim como em condições degenerativas de etiologias variadas que envolvam alteração progressiva do EPR como, por exemplo, coriorretinopatia serosa central e retinopatia por cloroquina (Figura 6). 4,38,39 


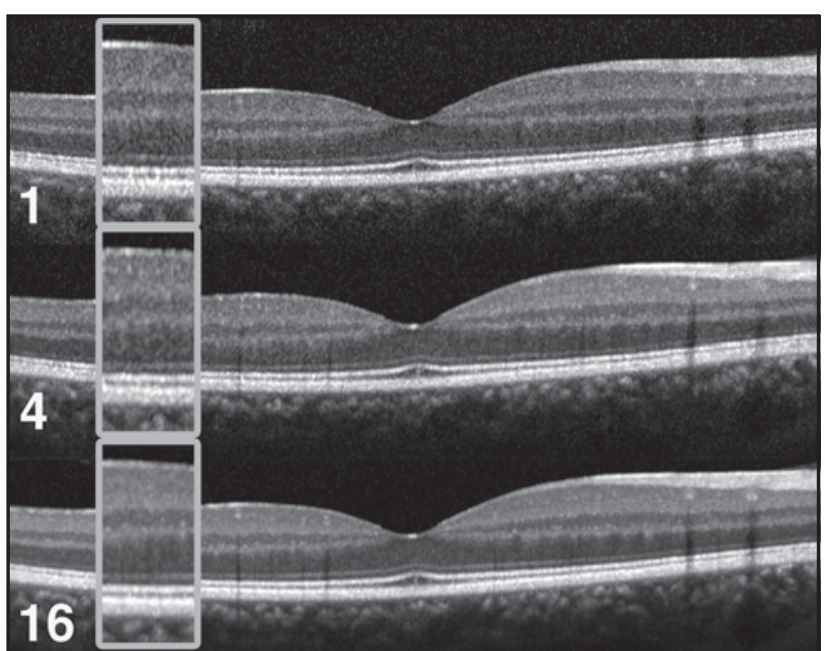

Figura 3. Aspecto da região macular normal por tomografia de coerência óptica espectral obtidos com o Spectralis ${ }^{\circledast}$ HRA+OCT utilizando módulo $A R T$ (automatic real time) em que vários $B$ scans obtidos da mesma região (por meio de um sistema de eyetracking) são processados para geração do B-scan final. Apesar de os 3 B-scans apresentarem a mesma "resolução" (axial), observem o aumento gradativo da qualidade (definição) em relação ao $B$-scan original (superior) a medida que mais imagens são utilizadas para a geração do tomograma final (meio, 4 B-scans; inferior, 16 B-scans).

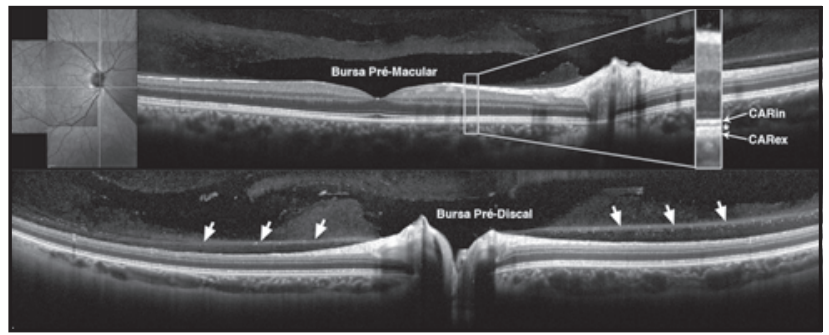

Figura 5. Avaliação por tomografia de coerência óptica espectral da mácula (superior) e região peridiscal (inferior) em fundo-deolho normal. Notem identificação da interface vítreo-retiniana (setas) e de peculiaridades morfológicas não identificáveis por outros recursos semiológicos, como as "bursas" pré-retinianas e uma nova camada de alta-refletividade (asterisco) entre a camada altamente refletiva interna (CARin) correspondente à junção dos segmentos interno e externo dos fotorreceptores e a camada altamente refletiva externa (CARex) correspondente ao complexo formado por epitélio pigmentado da retina e membrana de Bruch.

Portanto, a $F A F$ oferece informações que se somam aos achados da investigação de rotina por estudos angiográficos e tomográficos, contribuindo no diagnóstico e acompanhamento de patologias oculares em que o EPR pode estar direta ou indiretamente envolvido (Figura 7)..$^{33,34,40,41}$

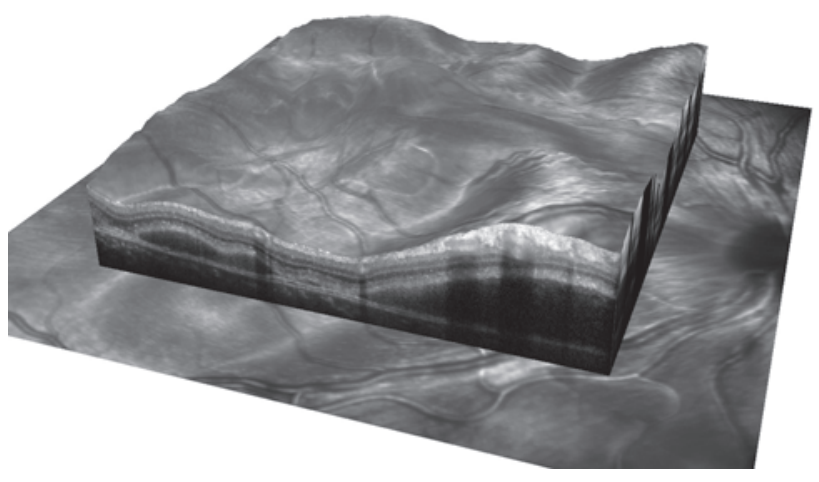

Figura 4. Reconstrução da topografia macular de um paciente com síndrome de Vogt-Koyanagi-Harada utilizando dados obtidos por tomografia de coerência óptica de alta-resolução e altavelocidade.

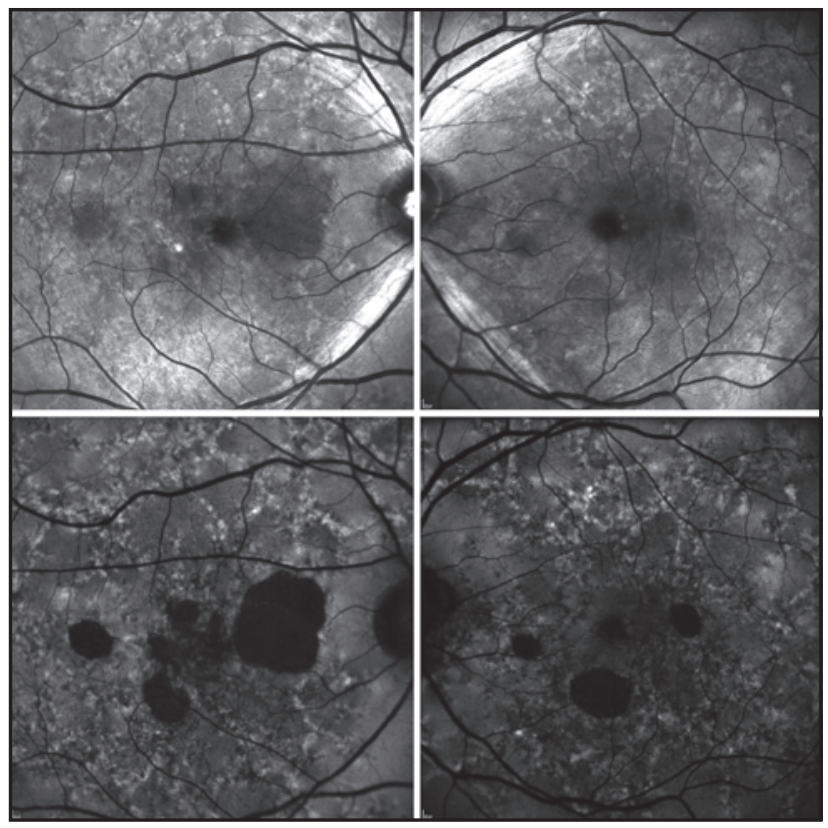

Figura 6. Avaliação ocular multimodal de paciente com Doença de Stargardt. A retinografia red-free (superior) mostra depósitos pisciformes difusos em ambos os olhos. A autofluorescência de fundo (fundus autofluorescence, $F A F$ ) (inferior) demonstra claramente lesões com aumento de autofluorescência bem como revela múltiplas áreas circunscritas de atrofia do EPR (com ausência de autofluorescência).

\section{Autofluorescência com Luz "Infravermelha" (near-Infrared Autofluorescence, nIA)}

A autofluorescência com luz "infravermelha" (near-infrared autofluorescence, nIA) contribui para a avaliação in vivo de melanina, outro pigmento característico do EPR. Durante a $n I A$ com o $c S L O$, a retina é 


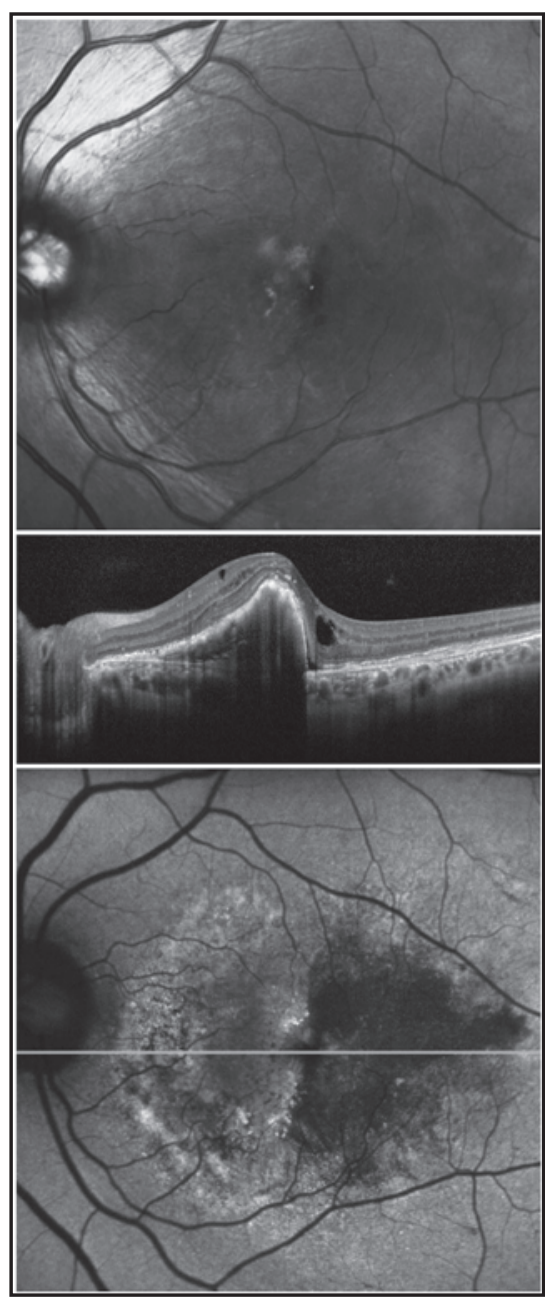

Figura 7. Avaliação ocular multimodal de paciente com neovascularização de coróide. A retinografia redfree (superior) e a tomografia de coerência óptica espectral (meio) demonstram elevação da região macular à custa do descolamento do epitélio pigmentado da retina (EPR) e a presença de algum fluido intraretiniano. A autofluorescência de fundo (inferior) revela presença de extensa rotura do EPR associada (área com ausência de fluorescência na metade temporal macular).
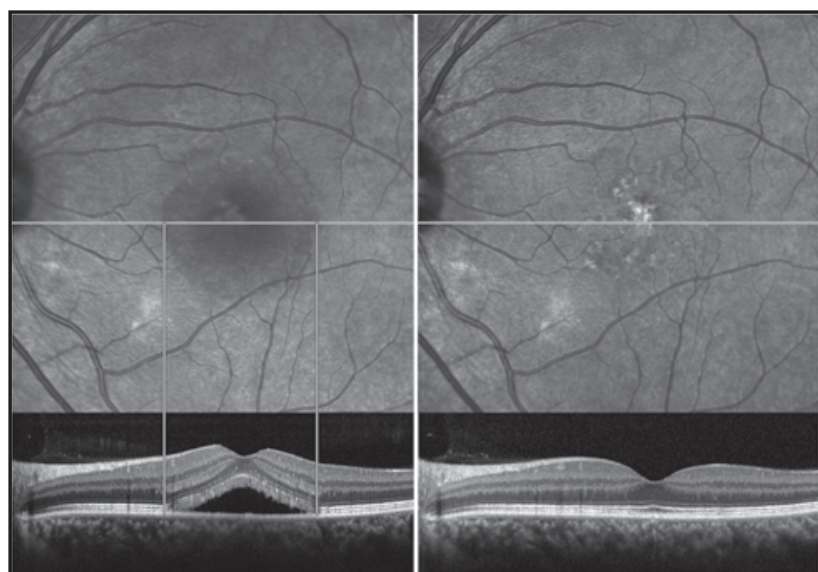

Figura 9. Reflectância com luz infravermelha (near-infrared reflectance, $n I R)$ e tomografia de coerência óptica espectral (spectral optical coherence tomography, SOCT) de paciente com coriorretinopatia serosa central. Na apresentação do quadro (esquerda) a área de elevação retiniana aparece mais escura na $n I R$. Quatro meses após a apresentação (direita) o SOCT demonstra restabelecimento da arquitetura macular sem alterações retinianas evidentes; não obstante, a $n I R$ revela intensa atividade pigmentar na área previamente elevada pelo fluido sub-retiniano.

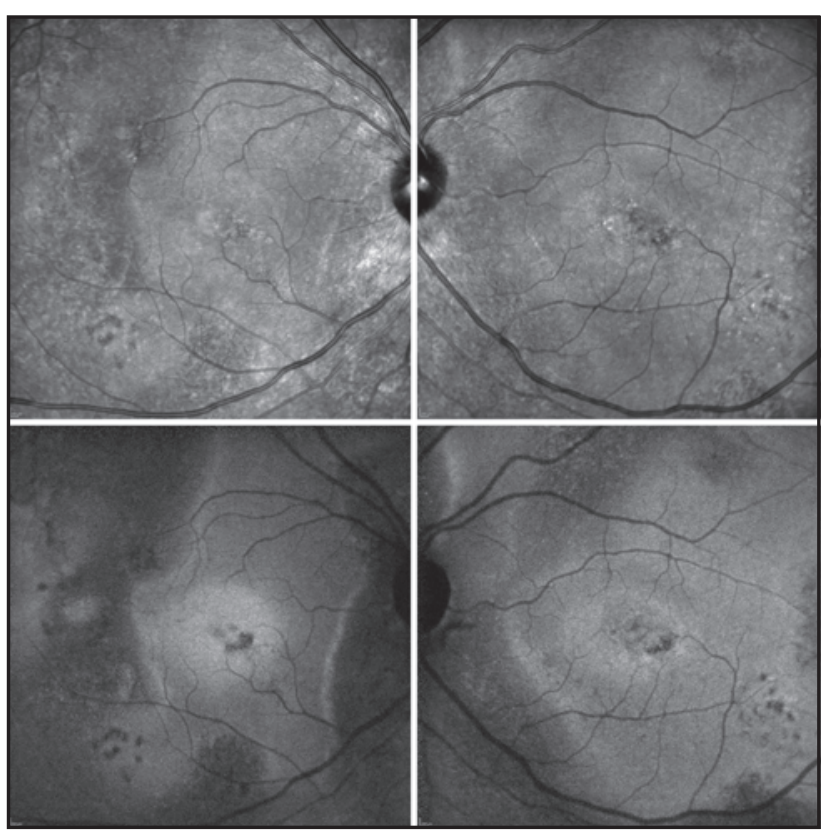

Figura 8. Avaliação ocular multimodal em paciente com coriorretinopatia serosa central crônica. Enquanto a reflectância com luz "infravermelha" (near-infrared reflectance, $n I R)$ (superior) mostra alterações pigmentares inespecíficas, a autofluorescência com luz infravermelha (near-infrared autofluorescence, $n I A)$ (inferior) delineia claramente as áreas de alteração pigmentar no pólo posterior.

iluminada com comprimento de onda de $787 \mathrm{~nm}$, e o filtro de barreira é idêntico ao utilizado para angiografia com indocianina verde. ${ }^{16}$ Contudo, a imagem do fundo ocular obtida é de limitada qualidade.

Os achados da $n I A$ são diferentes dos achados com autofluorescência de fundo padrão $(F A F)$. Evidências indicam que a distribuição da $n I A$ se correlaciona diretamente à distribuição da melanina no fundo ocular, com contribuições do EPR e da coróide. ${ }^{16,17,42,43}$ Anormalidades de $n I A$ foram reportadas em pacientes com anormalidades genéticas ou degenerativas do EPR, como Doença de Stargardt, retinite pigmentosa, degeneração macular relacionada à idade, retinopatia por cloroquina e coriorretinopatia serosa central (Figura 8). ${ }^{16,17,43,44}$

\section{Reflectância com Luz "Infravermelha" (near-Infrared Reflectance, $n I R)$}

A reflectância com iluminação "infravermelha" (near-infrared reflectance, nIR) permite a documentação de estruturas retinianas profundas e sub-retinianas, com resolução e contraste superiores às técnicas de imagem rotineiras. ${ }^{45}$ As imagens de $n I R$ são obtidas pelo cSLO com estímulo luminoso de $815 \mathrm{~nm}$. ${ }^{16}$

Apenas sistemas ópticos confocais permitem o 


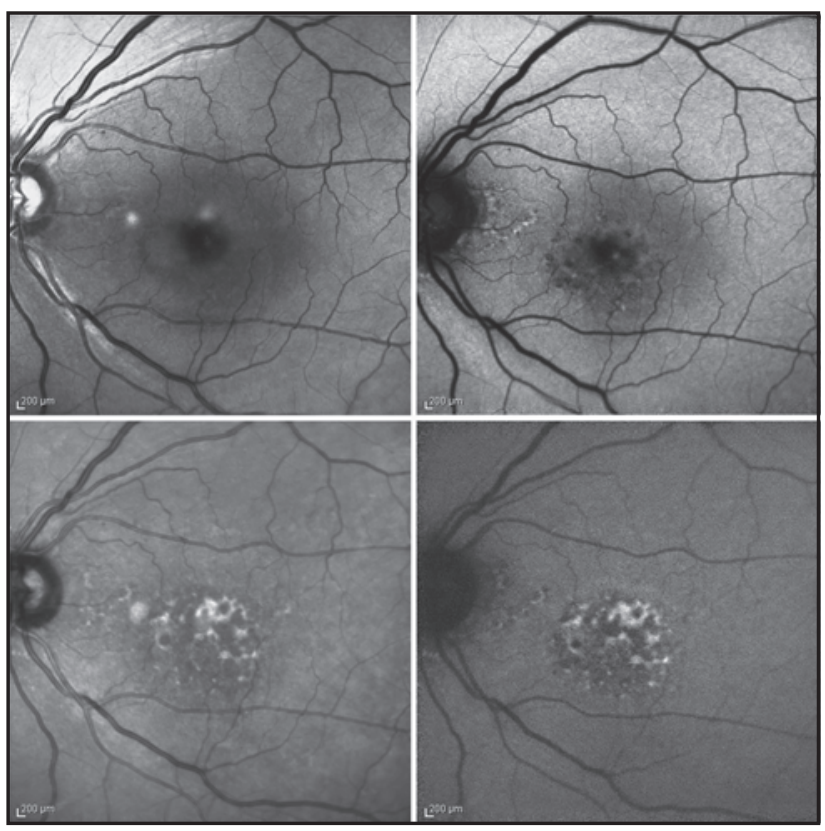

Figura 10. Avaliação ocular multimodal de paciente com coriorretinopatia serosa central crônica. A retinografia red-free (superior esquerda) sugere alteração topográfica na região macular compatível com presença de fluido sub-retiniano. Enquanto os achados de autofluorescência de fundo (fundus autofluorescence, $F A F$ ) (superior direita) são inespecíficos neste caso, a reflectância com luz "infravermelha" (near-infrared reflectance, $n I R$ ) (inferior esquerda) revela alterações pigmentares não evidentes ao exame clínico. A comparação entre a $n I R$ e a autofluorescência com luz "infravermelha" (near-infrared autofluorescence, $n I A$ ) (inferior direita) sugere que parte da fluorescência emitida com luz "infravermelha" possa ser pseudofluorescência.

registro adequado por $n I R$ por rejeitarem a luz difundida. A câmera de fundo convencional ("retinógrafo"), ao contrário, não consegue separar a luz refletida da luz difundida. A imagem obtida por $n I R$, por conseguinte, é ultimamente determinada pela reflexão e absorção de luz pelas diferentes camadas do tecido iluminado. A região foveal aparece mais "clara", decorrente da menor absorção de luz "infravermelha" pelos pigmentos maculares. Depósitos sub-retinianos como drusas, quando próximos ao plano de foco, são observados "claros" e obscurecem parcialmente os vasos da coróide. De maneira similar, cicatrizes sub-retinianas também refletem a luz "infravermelha". Camadas de fluido denso ou túrbido, ao contrário, não transmitem a luz lateralmente ou para planos mais profundos; conseqüentemente, áreas de descolamento do EPR (seroso ou serohemorrágico) aparecem "escuras" na imagem por $n I R$ (Figura 9). ${ }^{45,46}$

Portanto, a $n I R$ é especialmente valiosa na investigação de patologias que envolvam a membrana de Bruch ou que apresentem depósitos retinianos profundos, degeneração macular relacionada à idade ou estri- as angióides. ${ }^{1,16,47}$ Pode ainda, potencialmente, identificar patologias sub-clínicas, mesmo na presença de alguma opacidade de meios como esclerose lenticular moderada ou hemorragia vítrea leve, ou revelar drusas indetectáveis pelo exames convencionais. ${ }^{45}$ Seu uso como ferramenta auxiliar na monitorização de tratamentos da região macular vem também sendo investigada. ${ }^{17}$

\section{Avaliação Ocular Multimodal (Multimodal Ocular/Fundus Imaging)}

O registro angiográfico simultâneo de fluoresceína e indocianina verde foi proposto há várias décadas, ${ }^{48}$ tendo sido incorporado à rotina clínica apenas recentemente, quando os $c S L O$ se tornaram comercialmente disponíveis. ${ }^{49-51}$ Adicionalmente, por registrar apenas os feixes de luz confocal e suprimir a luz difundida, os sistemas de $c S L O$ são capazes de documentar um plano específico do tecido retiniano com contraste e detalhes impossíveis de serem obtidos presentemente com as câmeras de fundo convencionais. ${ }^{52-56}$ Essa propriedade óptica, somada ao estímulo luminoso e registro de bandas de comprimento de onda específicos, permitem a documentação com $F A F$ e $n I R$ em alta-resolução. O registro de imagens por $n I A$, entretanto, ainda apresenta uma série de limitações. A definição da imagem obtida não é alta, em comparação com outros modos de imagem, e a interpretação dos achados fenotípicos ainda é em parte controversa. A correlação entre $n I R$ e $n I A$ indica que parte da fluorescência observada por luz "infravermelha" possa ser pseudofluorescência, com influência direta na imagem obtida (Figura 10). ${ }^{44}$

A alta definição da imagem obtida com os novos aparelhos de $O C T$ espectral tem levado alguns autores a comparar o corte seccional tomográfico ao corte histológico. ${ }^{9110,57-59}$ Não obstante, a correlação direta com a histologia do tecido ainda não é conhecida em todas as situações fisiológicas e patológicas, e comparações devem ainda ser vistas com algum ceticismo. As características físicas das diferentes camadas celulares da retina obviamente influenciam a reconstrução óptica da imagem tomográfica, e os artefatos resultantes não podem ser desprezados. ${ }^{7,60}$ Nesse sentido, a análise integrada de imagens multimodal tem se mostrado fundamental na interpretação dos achados morfológicos da retina neurossensorial e EPR. Considerando-se os princípios básicos de aquisição de imagem e processamento dos dados, variados aspectos de diferentes camadas da retina podem ser revelados. Os achados morfológicos com a investigação multimodal são de tal qualidade em definição, sensibilidade e especificidade, que permitem 
inferências funcionais sobre o tecido. Esta estratégia investigativa tem implicações de interesse clínico e científico. ${ }^{61-65}$ Além de contribuir para o diagnóstico de desordens heredodistróficas e degenerativas da retina e EPR bem como na monitorização de progressão da doença ou resposta terapêutica, estudos futuros determinarão o real papel da análise integrada de imagens multimodal no entendimento de mecanismos fisiopatológicos da retina.

\section{Abstract}

Optical coherence tomography was progressively incorporated to the contemporary diagnostic arsenal in Ophthalmology, playing a crucial role in the diagnosis and management of eye diseases, particularly in the specialty of retina and vitreous. The commercial availability of the new generation of devices, coined "spectral" optical coherence tomography, which is based in a distinct physical concept that permits highspeed image acquisition, launched a new era for this investigative ancillary tool. In addition, the recent combination of this new technology with a confocal scanning laser ophthalmoscope has permitted the acquisition of tomographic images driven by different imaging modalities simultaneously (fundus autofluorescence, near-infrared reflectance, and fluorescein or indocyanine-green angiographies). Multimodal fundus imaging permits a reliable and detailed correlation between the morphological findings of the retina or retinal pigment epithelium and angiographic studies or fundus autofluorescence, leading to valuable insights about retina physiology. In this article, we briefly discuss possible practical implications of this new diagnostic modality for the retina specialist.

Keywords: Angiography; Retinal pigment epithelium; Tomography, optical coherence/methods; Fluorescence; Spectroscopy, Fourier transform infrared; Laser; Retina

\section{REFERÊNCIAS}

1. Charbel Issa P, Finger RP, Holz FG, Scholl HP. Multimodal imaging including spectral domain OCT and confocal near infrared reflectance for characterisation of outer retinal pathology in pseudoxanthoma elasticum. Invest Ophthalmol VisSci. 2009 Jun 24.[Epub ahead of print]

2. Brinkmann CK, Wolf S, Wolf-Schnurrbusch UE. Multimodal imaging in macular diagnostics: combined OCT-SLO improves therapeutical monitoring. Graefes Arch Clin Exp Ophthalmol. 2008;246(1):9-16.
3. Charbel Issa P, Foerl M, Helb HM, Scholl HP, Holz FG. Multimodal fundus imaging in foveal hypoplasia: combined scanning laser ophthalmoscope imaging and spectral-domain optical coherence tomography. Arch Ophthalmol. 2008;126(10):1463-5.

4. Spaide RF, Klancnik JM Jr. Fundus autofluorescence and central serous chorioretinopathy. Ophthalmology. 2005;112(5):825-33.

5. Spaide RF, Noble K, Morgan A, Freund KB. Vitelliform macular dystrophy. Ophthalmology. 2006;113(8):1392-400.

6. de Boer JF, Cense B, Park BH, Pierce MC, Tearney GJ, Bouma BE. Improved signal-to-noise ratio in spectral-domain compared with time-domain optical coherence tomography. Opt Lett. 2003;28(21):2067-9.

7. Costa RA, Skaf M, Melo LA Jr, Calucci D, Cardillo JA, Castro JC, et al. Retinal assessment using optical coherence tomography. Prog Retin Eye Res. 2006;25(3):325-53.

8. Drexler W, Sattmann H, Hermann B, Ko TH, Stur M, Unterhuber A, et al. Enhanced visualization of macular pathology with the use of ultrahigh-resolution optical coherence tomography. Arch Ophthalmol. 2003;121(5):695-706.

9. Wojtkowski M, Srinivasan V, Fujimoto JG, Ko T, Schuman JS, Kowalczyk A, et al. Three-dimensional retinal imaging with high-speed ultrahigh-resolution optical coherence tomography. Ophthalmology. 2005;112(10):1734-46.

10. Srinivasan VJ, Wojtkowski M, Witkin AJ, Duker JS, Ko TH, Carvalho M, et al. High-definition and 3-dimensional imaging of macular pathologies with high-speed ultrahigh-resolution optical coherence tomography. Ophthalmology. 2006;113(11):2054.e1-14.

11. Delori FC, Dorey CK, Staurenghi G, Arend O, Goger DG, Weiter JJ. In vivo fluorescence of the ocular fundus exhibits retinal pigment epithelium lipofuscin characteristics. Invest Ophthalmol Vis Sci. 1995 Mar;36(3):718-29.

12. Delori FC, Staurenghi G, Arend O, Dorey CK, Goger DG, Weiter JJ. In vivo measurement of lipofuscin in Stargardt's disease-Fundus flavimaculatus. Invest Ophthalmol Vis Sci. 1995;36(11):2327-31.

13. Delori FC, Fleckner MR, Goger DG, Weiter JJ, Dorey CK. Autofluorescence distribution associated with drusen in agerelated macular degeneration. Invest Ophthalmol Vis Sci. 2000;41(2):496-504.

14. Delori FC, Goger DG, Dorey CK. Age-related accumulation and spatial distribution of lipofuscin in RPE of normal subjects. Invest Ophthalmol Vis Sci. 2001;42(8):1855-66.

15. Schmitz-Valckenberg S, Holz FG, Bird AC, Spaide RF. Fundus autofluorescence imaging: review and perspectives. Retina. 2008;28(3):385-409.

16. Kellner S, Kellner U, Weber BH, Fiebig B, Weinitz S, Ruether K. Lipofuscin-and melanin-related fundus autofluorescence in patients with ABCA4-associated retinal dystrophies. Am J Ophthalmol. 2009;147(5):895-902, 902.e1.

17. Kellner U, Kellner S, Weber BH, Fiebig B, Weinitz S, Ruether K. Lipofuscin-and melanin-related fundus autofluorescence visualize different retinal pigment epithelial alterations in patients with retinitis pigmentosa. Eye. 2009;23(6):1349-59.

18. Lois N, Halfyard AS, Bird AC, Holder GE, Fitzke FW. Fundus autofluorescence in Stargardt macular dystrophy-fundus flavimaculatus. Am J Ophthalmol. 2004;138(1):55-63.

19. Feeney L. Lipofuscin and melanin of human retinal pigment epithelium. Fluorescence, enzyme cytochemical, and ultrastructural studies. Invest Ophthalmol Vis Sci. 1978;17(7):583-600. 
20. Feeney-Burns L, Berman ER, Rothman H. Lipofuscin of human retinal pigment epithelium. Am J Ophthalmol. 1980;90(6):783-91.

21. Feeney-Burns L, Hilderbrand ES, Eldridge S. Aging human RPE: morphometric analysis of macular, equatorial, and peripheral cells. Invest Ophthalmol Vis Sci. 1984;25(2):195-200.

22. Eldred GE. Age pigment structure. Nature. 1993;364(6436):396.

23. Boulton M, Dayhaw-Barker P. The role of the retinal pigment epithelium: topographical variation and ageing changes. Eye. 2001;15(Pt 3):384-9.

24. Shamsi FA, Boulton M. Inhibition of RPE lysosomal and antioxidant activity by the age pigment lipofuscin. Invest Ophthalmol Vis Sci. 2001;42(12):3041-6.

25. Holz FG, Schütt F, Kopitz J, Eldred GE, Kruse FE, Völcker $\mathrm{HE}$, et al. Inhibition of lysosomal degradative functions in RPE cells by a retinoid component of lipofuscin. Invest Ophthalmol Vis Sci. 1999;40(3):737-43.

26. Schütt F, Davies S, Kopitz J, Holz FG, Boulton ME. Photodamage to human RPE cells by A2-E, a retinoid component of lipofuscin. Invest Ophthalmol Vis Sci. 2000;41(8):2303-8.

27. Sparrow JR, Zhou J, Ben-Shabat S, Vollmer H, Itagaki Y, Nakanishi K. Involvement of oxidative mechanisms in bluelight-induced damage to A2E-laden RPE. Invest Ophthalmol Vis Sci. 2002;43(4):1222-7.

28. Sparrow JR, Boulton M. RPE lipofuscin and its role in retinal pathobiology. Exp Eye Res. 2005;80(5):595-606.

29. Sparrow JR, Cai B, Jang YP, Zhou J, Nakanishi K. A2E, a fluorophore of RPE lipofuscin, can destabilize membrane. Adv Exp Med Biol. 2006;572:63-8.

30. Kaemmerer E, Schutt F, Krohne TU, Holz FG, Kopitz J. Effects of lipid peroxidation-related protein modifications on RPE lysosomal functions and POS phagocytosis. Invest Ophthalmol Vis Sci. 2007;48(3):1342-7.

31. Wassell J, Davies S, Bardsley W, Boulton M. The photoreactivity of the retinal age pigment lipofuscin. J Biol Chem. 1999;274(34):23828-32.

32. Holz FG, Bellman C, Staudt S, Schütt F, Völcker HE. Fundus autofluorescence and development of geographic atrophy in age-related macular degeneration. Invest Ophthalmol Vis Sci. 2001;42(5):1051-6.

33. Einbock W, Moessner A, Schnurrbusch UEK, Holz FG, Wolf S. For the FAM Study Group. Changes in fundus autofluorescence in patients with age-related maculopathy. Correlation to visual function: a prospective study. Graefes Arch Clin Exp Ophthalmol. 2005;243:300-305.

34. Holz FG, Bindewald-Wittich A, Fleckenstein M, Dreyhaupt J, Scholl HP, FAM-Study Group, et al. Progression of geographic atrophy and impact of fundus autofluorescence patterns in age-related macular degeneration. Am J Ophthalmol. 2007;143(3):463-72.

35. Eagle RC Jr, Lucier AC, Bernardino VB Jr, Yanoff M. Retinal pigment epithelial abnormalities in fundus flavimaculatus: a light and electron microscopic study. Ophthalmology. 1980;87(12):1189-200.

36. Gerth C, Andrassi-Darida M, Bock M, Preising MN, Weber BH, Lorenz B. Phenotypes of 16 Stargardt macular dystrophy/fundus flavimaculatus patients with known ABCA4 mutations and evaluation of genotype-phenotype correlation. Graefes Arch Clin Exp Ophthalmol. $2002 ; 240(8): 628-38$.
37. Cideciyan AV, Swider M, Aleman TS, Sumaroka A, Schwartz SB, Roman MI, et al. ABCA4-associated retinal degenerations spare structure and function of the human parapapillary retina. Invest Ophthalmol Vis Sci. 2005;46(12):4739-46.

38. Kellner U, Renner AB, Tillack H. Fundus autofluorescence and mfERG for early detection of retinal alterations in patients using chloroquine/hydroxychloroquine. Invest Ophthalmol Vis Sci. 2006;47(8):3531-8.

39. Spaide R. Autofluorescence from the outer retina and subretinal space: hypothesis and review. Retina. 2008;28(1):5-35.

40. Bindewald A, Schmitz-Valckenberg S, Jorzik JJ, DolarSzczasny J, Sieber H, Keilhauer C, et al. Classification of abnormal fundus autofluorescence patterns in the junctional zone of geographic atrophy in patients with age related macular degeneration. $\mathrm{Br} \mathrm{J}$ Ophthalmol. 2005;89(7):874-8.

41. Sallo FB, Rechtman E, Peto T, Stanescu-Segall D, Vogt G, Bird $\mathrm{AC}$, et al. Functional aspects of drusen regression in age-related macular degeneration. Br J Ophthalmol. 2009;93(10):1345-50.

42. Weiter JJ, Delori FC, Wing GL, Fitch KA. Retinal pigment epithelial lipofuscin and melanin and choroidal melanin in human eyes. Invest Ophthalmol Vis Sci. 1986 ;27(2):145-52.

43. Keilhauer CN, Delori FC. Near-infrared autofluorescence imaging of the fundus: visualization of ocular melanin. Invest Ophthalmol Vis Sci. 2006;47(8):3556-64.

44. Weinberger AW, Lappas A, Kirschkamp T, Mazinani BA, Huth JK, Mohammadi B, et al. Fundus near infrared fluorescence correlates with fundus near infrared reflectance. Invest Ophthalmol Vis Sci. 2006;47(7):3098-108.

45. Elsner AE, Burns SA, Weiter JJ, Delori FC. Infrared imaging of sub-retinal structures in the human ocular fundus. Vision Res. 1996;36(1):191-205.

46. Delori FC, Pflibsen KP. Spectral reflectance of the human ocular fundus. Appl Opt. 1989;28:1061-1077.

47. Sayanagi K, Sharma S, Kaiser PK. Spectral domain optical coherence tomography and fundus autofluorescence findings in pseudoxanthoma elasticum. Ophthalmic Surg Lasers Imaging. 2009;40(2):195-7.

48. Flower RW, Hochheimer BF. A clinical technique and apparatus for simultaneous angiography of the separate retinal and choroidal circulations. Invest Ophthalmol. 1973;12(4):248-61.

49. Bartsch DU, Weinreb RN, Zinser G, Freeman WR. Confocal scanning infrared laser ophthalmoscopy for indocyanine green angiography. Am J Ophthalmol. 1995 ;120(5):642-51.

50. Holz FG, Bellmann C, Rohrschneider K, Burk RO, Völcker HE. Simultaneous confocal scanning laser fluorescein and indocyanine green angiography. Am J Ophthalmol. 1998;125(2):227-36.

51. Freeman WR, Bartsch DU, Mueller AJ, Banker AS, Weinreb RN. Simultaneous indocyanine green and fluorescein angiography using a confocal scanning laser ophthalmoscope. Arch Ophthalmol. 1998;116(4):455-63.

52. Fercher AF, Hitzenberger CK, Kamp G, Elzaiat SY. Measurement of intraocular distances by backscattering spectral interferometry. Opt Commun.1995; 15;117(1/2):43-8.

53. von Rückmann A, Fitzke FW, Bird AC. Distribution of fundus autofluorescence with a scanning laser ophthalmoscope. $\mathrm{Br} \mathrm{J}$ Ophthalmol. 1995;79(5):407-12.

54. Webb RH, Hughes GW, Pomerantzeff O. Flying spot TV ophthalmoscope. Appl Opt. 1980;19(17):2991-7.

55. Webb RH, Hughes GW, Delori FC. Confocal scanning laser ophthalmoscope. Appl Opt. 1987;26(8):1492-9. 
56. Jorzik JJ, Bindewald A, Dithmar S, Holz FG. Digital simultaneous fluorescein and indocyanine green angiography, autofluorescence, and red-free imaging with a solid-state laser-based confocal scanning laser ophthalmoscope. Retina.2005;25(4):405-16.

57. Zawadzki RJ, Jones SM, Olivier SS, Zhao M, Bower BA, Izatt JA, et al.Adaptive-optics optical coherence tomography for high-resolution and high-speed $3 \mathrm{D}$ retinal in vivo imaging. Opt Express. $2005 ; 13(21): 8532-46$.

58. Srinivasan VJ, Monson BK, Wojtkowski M, Bilonick RA, Gorczynska I, Chen R, et al. Characterization of outer retinal morphology with high-speed, ultrahigh-resolution optical coherence tomography. Invest Ophthalmol Vis Sci. 2008;49(4):1571-9.

59. Drexler W, Fujimoto JG. State-of-the-art retinal optical coherence tomography. Prog Retin Eye Res. 2008;27(1):45-88.

60. Costa RA. Evaluation of image artifact produced by optical coherence tomography of retinal pathology. Am J Ophthalmol. 2005;140(2):349-50.

61. Lamb LE, Simon JD. A2E: a component of ocular lipofuscin. Photochem Photobiol. 2004;79(2):127-36.

62. Wang Z, Keller LM, Dillon J, Gaillard ER. Oxidation of A2E results in the formation of highly reactive aldehydes and ketones. Photochem Photobiol. 2006;82(5):1251-7.
63. Zhou J, Jang YP, Kim SR, Sparrow JR. Complement activation by photooxidation products of A2E, a lipofuscin constituent of the retinal pigment epithelium. Proc Natl Acad Sci U S A. 2006;103(44):16182-7.

64. Lakkaraju A, Finnemann SC, Rodriguez-Boulan E. The lipofuscin fluorophore $\mathrm{A} 2 \mathrm{E}$ perturbs cholesterol metabolism in retinal pigment epithelial cells. Proc Natl Acad Sci U S A. 2007;104(26):11026-31.

65. Wang Z, Dillon J, Gaillard ER. Antioxidant properties of melanin in retinal pigment epithelial cells. Photochem Photobiol. 2006;82(2):474-9.

Endereço para correspondência:

Rogério A. Costa.

Centro Brasileiro de Ciências Visuais

Rua dos Otoni, 881 conjunto 1001, Santa Efigênia

CEP 30.150-270 - Belo Horizonte (MG) - Brasil.

Telefone/fax: (31) 3274-4001

Email: roger.retina@globo.com 